\title{
ACUTE AND EMERGENCY DEPARTMENT VISITS OF PATIENTS WITH DEMENTIA AT A UNIVERSITY HOSPITAL IN NORTHWESTERN GREECE: REASON,DIAGNOSIS AND OUTCOME.
}

Christopoulou F. (1), Tatsioni A.(2), Florentin M.(1), Gatsiou K.(3), Peritogiannis V. (4)

(1)Consultant of Internal Medicine, Emergency Department University Hospital of loannina

(2)Ass. Prof. and Head Research Unit for General Medicine and Primary Health Care Faculty of Medicine, School of Health Sciences, University of loannina (3)Psychiatric Trainee, Psychiatric Clinic University Hospital of loannina

(4)Psychiatrist, Hellenic Open University Tutor

Introduction: The population is aging worldwide, resulting in high incidence of comorbidities like dementia, which leads to severe cognitive and physical impairment.

Background and Aims: Patients with dementia are prone to long hospitalizations, develop complications, have worse prognosis and outcome. However, little is known regarding the actual indications and the necessity for Acute and Emergency department (AnE) visits. The purpose of this study was to assess the cause, the comorbidities and the final outcomes of patients with dementia visiting the AnE.

Material and Methods: This retrospective matched casecontrol study was conducted on a sample of 100 patients with dementia aged $>65$ years that visited the AnE of the University Hospital of loannina, over a four month period. Inclusion criteria were age $>65$ and history of dementia.

Data was collected from the hospital electronic health records respecting patients confidentiality policy. Each case was matched for sex and age, with a control patient without dementia that visited the AnE on the same day. They were assessed regarding dementia diagnosis, place of stay, existence of caregiver, cause of visit, comorbidities, diagnosis, hospitalization and length of hospital stay.

\begin{tabular}{|l|l|l|}
\hline VARIABLES & DEMENTIA & COMTROLS \\
\hline AGE & $85(80-88)$ & $83(79-86)$ \\
\hline SEX & & \\
\hline MALE & $37(37 \%)$ & $37(37 \%)$ \\
\hline FEMALE & $63(63 \%)$ & $63(63 \%)$ \\
\hline CARERER & & \\
\hline NO DATA & $7(7 \%)$ & $58(58 \%)$ \\
\hline FORMAL & $19(19 \%)$ & - \\
\hline INFORMAL & $61(61 \%)$ & $9(9 \%)$ \\
\hline NO & $13(13 \%)$ & $33(33 \%)$ \\
\hline PLACE & & \\
\hline NO DATA & $15(15 \%)$ & $12(12 \%)$ \\
\hline IOANNINA & $73(73 \%)$ & $75(75 \%)$ \\
\hline OUTSKIRTS & $12(12 \%)$ & $13(13 \%)$ \\
\hline COMORBIDITIES & $2(2-3)$ & $2(2-3)$ \\
\hline OUTCOME & & \\
\hline ADMISSION & $27(27 \%)$ & $45(45 \%)$ \\
\hline DISCHARGE & $73(73 \%)$ & $55(55 \%)$ \\
\hline $\begin{array}{l}\text { HOSPITALIZATION } \\
\text { DURATION }\end{array}$ & $6(4-9)$ & $5(3-8)$ \\
\hline $\begin{array}{l}\text { DEMENTIA } \\
\text { DIAGNOSIS }\end{array}$ & & \\
\hline NO DATA & $22(22 \%)$ & - \\
\hline $\begin{array}{l}\text { MEDICATIONS } \\
\text { (MEDS) }\end{array}$ & $57(57 \%)$ & - \\
\hline DOCTOR/MEDS & $15(15 \%)$ & - \\
\hline DOCTOR & $6(6 \%)$ & - \\
\hline \multicolumn{2}{|l}{} & \\
\hline
\end{tabular}

\begin{tabular}{|c|c|c|}
\hline $\begin{array}{l}\text { CAUSE } \\
\text { (MOST } \\
\text { COMMON) }\end{array}$ & $\begin{array}{l}\text { DEMENT } \\
\begin{array}{l}\text { IA }(n= \\
100, \%)\end{array}\end{array}$ & $\begin{array}{l}\text { CONTRO } \\
\text { LS (n= } \\
\underline{100, \%)}\end{array}$ \\
\hline FEVER & 31 (31\%) & $13(13 \%)$ \\
\hline FALL & $14(14 \%)$ & $3(3 \%)$ \\
\hline $\begin{array}{l}\text { GENERAL } \\
\text { DETERIORAT } \\
\text { ION }\end{array}$ & $13(13 \%)$ & 7 (7\%) \\
\hline $\begin{array}{l}\text { REDUSED } \\
\text { LEVEL OF } \\
\text { CONCIOUSN } \\
\text { ESS }\end{array}$ & $7(7 \%)$ & 7 (7\%) \\
\hline $\begin{array}{l}\text { NEUROLOGI } \\
\text { CAL- OTHER }\end{array}$ & $7(7 \%)$ & $12(12 \%)$ \\
\hline $\begin{array}{l}\text { GASTRINTES } \\
\text { TINAL (GI) }\end{array}$ & $6(6 \%)$ & $16(16 \%)$ \\
\hline $\begin{array}{l}\text { GI } \\
\text { BLEEDING }\end{array}$ & $6(6 \%)$ & $4(4 \%)$ \\
\hline DYSPNEA & $4(4 \%)$ & $10(10 \%)$ \\
\hline DIZINESS & $4(4 \%)$ & 9 (9\%) \\
\hline ANAEMIA & $4(4 \%)$ & $2(2 \%)$ \\
\hline
\end{tabular}

References 